\author{
Tanja Werner ${ }^{\mathrm{a}}$ \\ Uwe Gröber ${ }^{b}$ \\ Klaus Kisters ${ }^{b, c}$
}

\section{Bedeutung der Elektrolyte und Vitamine bei Hypertonie}

\section{Einleitung}

Die primäre oder essentielle Hypertonie (Blutdruck $\geq 140$ und/oder $\geq 90$ $\mathrm{mm} \mathrm{Hg}$ ) ist die häufigste internistische Erkrankung in Deutschland mit 20-30 Millionen betroffenen Bundesbürgern. Weltweit leiden ca. 25\% der Bevölkerung an Bluthochdruck, mit steigender Tendenz. Häufig fehlen klinische Symptome, sodass es eine hohe Dunkelziffer bei dieser Erkrankung gibt. Die Hypertonie stellt den Risikofaktor Nr. 1 für Herz-Kreislauf-Erkrankungen dar und ist somit eine wesentliche Determinante der häufigsten Todesursachen im Erwachsenenalter. Besonders bei langjährig bestehendem Hypertonus können schwere Folgeerkrankungen wie Herzinfarkt, Herzschwäche oder Schlaganfall auftreten [1].

Für die Entstehung einer Hypertonie ist das Zusammenwirken von Genetik, Alter, Geschlecht und verschiedenen ungünstigen Ernährungs- und Lebensbedingungen verantwortlich. Dazu zählen Übergewicht, hoher Kochsalz- und Alkoholkonsum, Bewegungsmangel und Stress. In den seltensten Fällen ist Bluthochdruck die Folge von anderen Erkrankungen. Durch die Umstellung von Ernährungs- und Lebensgewohnheiten sowie eine konsequente medikamentöse Therapie kann der Risikofaktor entscheidend beeinflusst werden.

Protina, Ismaning, Deutschland; ${ }^{\mathrm{b}}$ Akademie für Mikronährstoffmedizin, Essen, Deutschland; ' ${ }^{\mathrm{c}}$ Medizinische Klinik I, St. Anna-Hospital, Herne, Germany
Die wissenschaftlichen Erkenntnisse der letzten Jahre haben gezeigt, dass auch hierbei Elektrolyte und Vitamine einen grossen Stellenwert bei der Behandlung einer essentiellen Hypertonie haben. Neben der medikamentösen Behandlung bei Hypertonie kommt somit auch den nichtmedikamentösen Therapieformen eine grosse Bedeutung zu. Die Herausforderung, hier Therapieansätze für Elektrolyte und Vitamine als Behandlungskonzepte für diese Erkrankung zu finden, ist gross.

\section{Elektrolyte und Hypertonie}

Mikro- und Makronährstoffdefizite bzw. Störungen im Elektrolythaushalt sind bei Patienten mit Hypertonie gut dokumentiert [2-4]. Antihypertensive Medikamente selbst spielen bei der Entstehung von Mineralstoffmängeln eine Rolle [5]. Besondere Bedeutung kommt weiterhin dem negativen Einfluss von Kochsalz (Natriumchlorid) auf die Hypertonie sowie dem positiven Effekt von Kalium und Magnesium zu. Auch niedrige Zink- und Kupferspiegel sowie eine Vielzahl an Vitaminen werden mit Bluthochdruck in Verbindung gebracht. Ebenso werden weitere Ernährungsfaktoren wie die Protein- und Ballaststoffzufuhr, Omega-3-Fettsäuren und Zucker diskutiert.

\section{Kochsalz}

Bereits vor 2300 Jahren wurde von einem chinesischen Arzt beobachtet, dass stark gesalzene Speisen den Puls hart machen und für Schlaganfälle mit Aphasie prädestinieren, also zu typischen Befunden bzw. Komplikationen der arteriellen Hypertonie führen können. In den letzten Jahrzehnten haben wissenschaftliche Arbeiten über die Bedeutung des Kochsalzentzugs für die Hochdrucktherapie berichtet. Zahlreiche Untersuchungen verschiedener Populationen mit unterschiedlichem Kochsalzkonsum ergeben eine auffällige Parallelität zwischen der Höhe des Kochsalzverbrauchs und der Hochdruckhäufigkeit. Heute gehört eine kochsalzarme Diät $(<6 \mathrm{~g} / \mathrm{Tag})$ zur Basistherapie jeder arteriellen Hypertonie. Dabei ist jedoch zu berücksichtigen, dass nur die Hälfte aller Hypertoniker salzsensitiv ist; d.h., nur bei diesen Patienten hat eine Kochsalzreduktion einen blutdrucksenkenden Effekt. Zusätzlich zeigen aktuelle Untersuchungen, dass eine sehr hohe, aber auch eine sehr niedrige Kochsalzzufuhr $(<2,5 \mathrm{~g} / \mathrm{Tag}) \mathrm{zu}$ einer erhöhten Mortalität führt [6].

Erhöhter Salzkonsum über Jahre und Jahrzehnte ist offensichtlich ein vaskulärer Risikofaktor, der zur vorzeitigen Alterung bzw. Versteifung der Gefässe und systolischer Blutdruckerhöhung führt und damit eine Kaskade von Folgeschäden in Gang setzt. Die nachlassende Windkesselfunktion der grossen Arterien durch erhöhte Gefässsteifigkeit führt letztlich über eine Augmentation des zentralen (aortalen) Blutdrucks als Folge der beschleunigten Pulswellenreflexion zum erhöhten Nachlass des Herzens, zu Linksherzhypertrophie und zu Herz-

\section{KARGER}

(c) 2017 S. Karger GmbH, Freiburg

Fax +497614520714
Prof. Dr. med. Klaus Kisters 
Tab. 1. Erhöhter Kochsalzverbrauch und Folgen

\section{Erhöhte Gefässsteifigkeit}

Verminderte Compliance der großen Gefässe

Erhöhtes Schlagvolumen

Verminderter diastolischer Fluss

Systolischer Blutdruckanstieg

Isolierte systolische Hypertonie

Erhöhte Pulswellengeschwindigkeit und verfrühte/ erhöhte Reflexion der Pulswelle

Augmentation des aortalen Blutdrucks, vermehrte Nachlast, erhöhte Ventrikellast

Linksherzhypertrophie

Verminderte diastolische Koronarperfusion

Myokardischämie

Herzinsuffizienz

Tab. 2. Effekte einer erhöhten Kochsalzzufuhr

Hypertrophie von Myokardzellen

Zunahme der linksventrikulären Muskelmasse

Erhöhte AT-1-Rezeptorenexpression im Herzmuskel

Hypertrophie von Gefässmuskelzellen

Abnahme der arteriellen Compliance

Erhöhung des Pulsdrucks

Endotheliale Dysfunktion

Verminderte Synthese von Stickoxid

Abschwächung des Barorezeptorenreflexes

Erhöhung der glomerulären Filtrationsrate (GFR)

Zunahme der Mikroalbuminurie

insuffizienz (Tab. 1). Klinische und experimentelle Untersuchungen haben gezeigt, dass zwischen Natriumchloridaufnahme und linksventrikulärer Muskelmasse eine positive Korrelation besteht. Umgekehrt nimmt unter Kochsalzbeschränkung bei Patienten mit essentieller Hypertonie die linksventrikuläre Muskelmasse wieder deutlich ab. Unabhängig von der Höhe des Blutdrucks ist eine erhöhte Kochsalzzufuhr mit einer Vergrösserung des Pulsdrucks verbunden. Hieraus resultiert eine verminderte Compliance der grossen arteriellen Gefässe, wodurch auch auf eine direkte Natriumwirkung an der Gefässwandstruktur geschlossen werden kann. Unter kochsalzreicher Kost steigt auch die glomeruläre Filtrationsrate an, die eine verstärkte Mikroalbuminurie begünstigt (Tab. 2).

In mehreren Studien wurde der Effekt einer Kochsalzreduktion sowohl
Tab. 3. Empfehlungen der Fachgesellschaften zur Kochsalzrestriktion

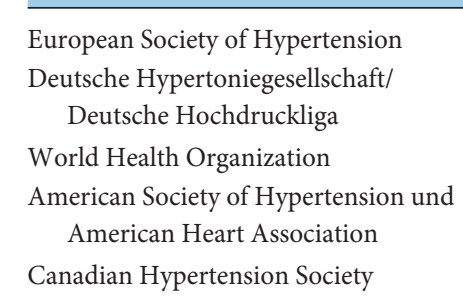

Zufuhr

$<6 \mathrm{~g}$

$<6 \mathrm{~g}$

$<5 \mathrm{~g}$

$<6$ g (empfohlen:

$\left.<3,8 \mathrm{~g} / \mathrm{Tag}^{\mathrm{a}}\right)$

$<6$ g (empfohlen:

$<3,0-3,8$ g/Tag $\left.{ }^{\mathrm{a}}\right)$

aAltersabhängige Empfehlung. auf den systolischen als auch auf den diastolischen Blutdruck bei Patienten nachgewiesen. In einer grossen Studie aus dem Jahr 1997 wurde in einem Crossover-Design für eine insgesamt vierwöchige salzarme Diät ein positiver Effekt einer Kochsalzrestriktion nachgewiesen. Gemessen wurde die Natriumausscheidung im 24-Stunden-Sammelurin. Bereits nach 2 Wochen salzarmer Diät kam es zu einer statistisch signifikanten Blutdrucksenkung, die auch nach 4 Wochen noch anhielt und dann nach dem Wechsel auf eine normale, also salzreiche Diät wieder einen Blutdruckanstieg auf Ausgangswerte zeigte. Diese Studie zeigte die Ergebnisse von 47 unbehandelten Senioren im Alter zwischen 60 und 78 Jahren mit einem Blutdruck zwischen 123 und 205 systolisch sowie 64 und 112 diastolisch. Eine durchschnittliche Reduktion um $83 \mathrm{mmol} \mathrm{Kochsalz/l} \mathrm{war} \mathrm{mit} \mathrm{einer}$ Blutdrucksenkung von 7,2 $\mathrm{mm} \mathrm{Hg}$ systolisch und 3,2 $\mathrm{mm} \mathrm{Hg}$ diastolisch assoziiert [7]. He und McGregor [8] konnten nachweisen, dass eine Reduktion der Kochsalzdiät über die empfohlene Höchstmenge hinaus bis zu 3 g/Tag mit einer weiteren Blutdrucksenkung assoziiert ist und prinzipiell in die Therapieempfehlung eingehen sollte. Dies berücksichtigt auch die aktuelle Empfehlung der Weltgesundheitsorganisation (WHO). Das relative Risiko für kardiovaskuläre Erkrankungen und Schlaganfall in Abhängigkeit von der Kochsalzzufuhr wurde ebenfalls untersucht. In einer Metaanalyse, die auf 13 Studien ba- siert, in denen der Einfluss der Kochsalzzufuhr auf den Blutdruck untersucht wurde, zeigte sich auch hier die Inzidenz kardiovaskulärer Erkrankungen reduziert. Hierbei sind 177000 Teilnehmer erfasst worden. Während der Beobachtungsdauer von 3,5-19 Jahren wurden mehr als 11000 vaskuläre Ereignisse dokumentiert. Eine grössere Kochsalzzufuhr war mit einem erhöhten Schlaganfallrisiko assoziiert. Auch kardiovaskuläre Erkrankungen im Allgemeinen zeigten eine statistisch grenzwertig signifikante Assoziation mit der Kochsalzzufuhr [9]. In einer grossen Studie an 19000 Krankenschwestern, die unter dem Namen Dash-Diät bekannt wurde, konnte gezeigt werden, dass über einen Zeitraum von 24 Jahren kardiovaskuläre Ereignisse einschliesslich Schlaganfall signifikant weniger in der mit Kochsalz am wenigsten belasteten Gruppe aufgetreten sind. Hieraus resultieren die Empfehlungen der Fachgesellschaften zur Kochsalzrestriktion bei Hypertonie (Tab. 3).

Auch der aktuelle Cochrane-Review [10] spricht für eine Reduktion des Kochsalzverbrauchs von 9-12 auf 5-6 g pro Tag, da sich innerhalb der Metaanalyse ein blutdrucksenkender Effekt und damit verbunden ein (vermutetes) niedrigeres Risiko für kardiovaskuläre Erkrankungen zeigt.

Um exaktere Empfehlungen und genauere Daten zu erhalten, sollten weiterführende Studien sowohl die Zeit bis zur maximalen Wirksamkeit als auch die Dosis-Wirkungs-Beziehung berücksichtigen, da diese inner- 
Tab. 4. Kaliumaufnahme: NHANES 2003-2008

Kochsalzzufuhr zu hoch bei Hypertonikern (ca. $60 \%>3000 \mathrm{mg} / \mathrm{Tag}$ )

Kaliumzufuhr erniedrigt bei Hypertonikern (ca. $2 \%>4700 \mathrm{mg} / \mathrm{Tag}$ )

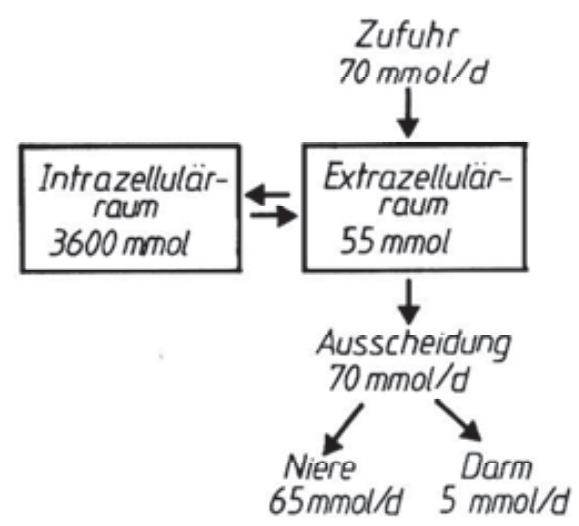

Abb. 1. Zufuhr, Ausscheidung und hauptsächliche Kompartimente des Kaliums.

halb der Metaanalysen zu Verzerrungen führen können. Ebenso sollte innerhalb der Studie die Differenzierung nach der individuellen Salzsensitivität erfolgen [6].

\section{Kalium}

Störungen im Kaliumstoffwechsel sind in der inneren Medizin bekanntermassen ein häufiges Problem. So haben sich Kaliummangelzustände bei Hypertonikern häufig als ungünstig erwiesen. Ein Kaliummangel tritt gelegentlich als Folge einer antihypertensiven Therapie, z.B. bei Diuretika-Einnahme, auf (Abb. 1). Weitere häufige Ursachen einer Hypokaliämie sind mangelnde Kaliumzufuhr, Kaliumverluste oder Kaliumverschiebungen in den Intrazellularraum. Tabelle 4 zeigt epidemiologische Daten bezüglich der Kochsalz- und Kaliumzufuhr.

Eine Vielzahl an Studien belegt den positiven Effekt einer Kaliumsupplementation auf den Blutdruck [11-13]. Die lineare Dosis-Wirkungs-Beziehung einer Kaliumeinnahme bei Blut-

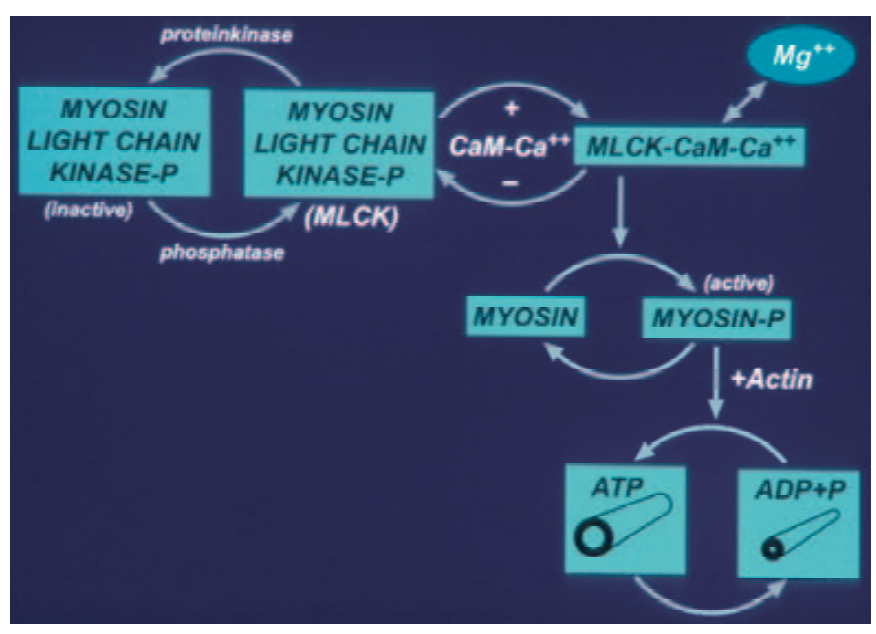

Abb. 2. Calcium-Magnesium-Antagonismus bei Hypertonie.

hochdruck zeigt sich durch eine blutdrucksenkende Wirkung von 1,0 mm $\mathrm{Hg}$ systolisch und 0,52 $\mathrm{mm} \mathrm{Hg}$ diastolisch pro $0,6 \mathrm{~g} /$ Tag mehr verzehrtem Kalium. Hierbei zeigte sich ebenso eine Reduktion des allgemeinen Mortalitätsrisikos um $20 \%$ je 1000 mg erhöhte Kaliumzufuhr.

\section{Magnesium}

Die Magnesiummangeltheorie bei Hypertonie wird durch neuere Studien zur Wirksamkeit von Magnesium untermauert. Magnesium ist für den Lebendorganismus ein essentieller Mineralstoff. In der Zelle ist Magnesium für die Aktivierung einer Vielzahl von Enzymen und Transportproteinen verantwortlich. Pathophysiologisch ist der Calcium-MagnesiumAntagonismus bei Hypertonie von wichtiger Bedeutung. Magnesium gilt als physiologischer Calcium-Antagonist. Hierbei kommt es zu Interaktionen zwischen Calcium-Magnesium im Bereich der Myosin-LeichtkettenKinase. Die ATP-abhängige Kontraktion der Myosinfilamente im Bereich der glatten Gefässmuskelzelle wird hierbei bei Magnesiummangel gestört und die Hypertonie dadurch verstärkt (Abb. 2) $[14,15]$.

In Untersuchungen von GuerreroRomero et al. [16] konnte gezeigt werden, dass sich bei gesunden Kindern mit niedrigem Magnesiumspiegel im
Alter statistisch signifikant häufiger eine Prähypertonie und Hypertonie entwickelt. Somit ist aus präventiven Gründen der Vermeidung einer Hypertonie ein Magnesiummangel zu verhindern. Eine orale Magnesiumsupplementation (300-1000 mg Magnesium täglich) hat sich bei Patienten mit Grenzwerthypertonie und Hypertonie als positiv erwiesen. Hierbei wurde ein statistisch signifikanter positiver Effekt sowohl auf den systolischen als auch auf den diastolischen Blutdruck beschrieben [14, 15]. Zusätzlich kommt es unter der Therapie mit Magnesium häufig zu einer Verbesserung der Endothelfunktion oder der Intima-Media-Dicke-Entstehung im Bereich der Halsschlagadern. Ebenso verstärkt Magnesium die Effektivität von antihypertensiven Medikamenten und ist als adjuvante Therapie bei Hypertonie empfehlenswert [17].

\section{Vitamin D}

In vielen Industrienationen ist ein Mangel an Vitamin D weit verbreitet, besonders in den Wintermonaten, wenn der UV-Index nicht ausreicht. Neben den bekannten Wirkungen auf den Knochenstoffwechsel schützt ein hoher Vitamin-D-Spiegel auch vor kardiovaskulären Erkrankungen, Diabetes mellitus und Hypertonie. Je niedriger die Vitamin-D-Konzentra- 

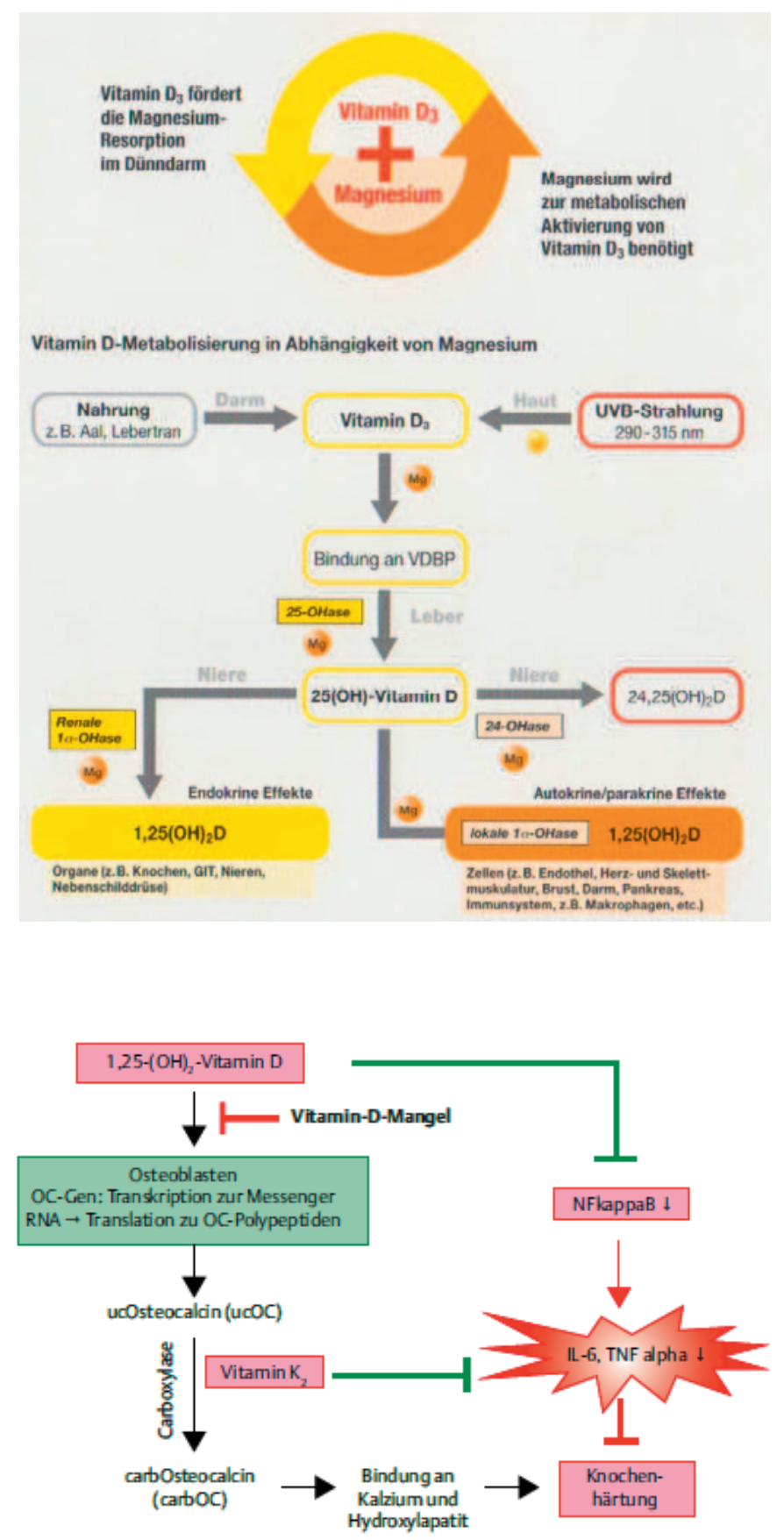

Abb. 3. Vitamin D und Magnesium.
Abb. 4. Vitamin D und $\mathrm{K}$. tion, desto höher ist das Risiko für Hypertonie $[18,19]$. Dennoch sind die Daten bezüglich einer Vitamin-DSupplementation bei Hypertonie kontrovers. In der DAYLIGHT-Studie wurde eine niedrige Vitamin-DDosierung (400 IE/Tag) gegenüber einer hochdosierten Gabe (4000 IE/ Tag) für 6 Monate bei Patienten mit Prähypertonie, Hypertonie Grad 1 und niedrigem Vitamin-D-Status getestet. Es konnte kein Unterschied hinsichtlich des 24-Stunden-Blutdrucks festgestellt werden [20]. In der placebokontrollierten Studie von Pilz et al. [21] hatte die Gabe von 2800 IE für 8 Wochen bei Patienten mit Hypertonie und niedrigen Vitamin-DSpiegeln ebenso keinen Einfluss auf den 24-Stunden-Blutdruck. Auch aktuelle Metaanalysen zeigen keine positiven Effekte einer Vitamin-D-Supplementation bei Hypertonie [22-24]. $\mathrm{Ob}$ die Patientenzahlen ausreichen und eine Supplementation nur im Falle von schwerem Vitamin-D-Mangel Effekte auf den Blutdruck zeigt, muss in weiteren Studien untersucht werden, damit eine abschliessende Empfehlung an Hypertonie-Patienten erfolgen kann.

Dokumentiert ist ein Zusammenhang zwischen 25-Hydroxy-VitaminD-Serumspiegel (25 OHD) und Mortalität [25]. Empfohlen werden ausreichend hohe 25-OHD-Spiegel, insbesondere bei älteren Menschen, zur Prävention von Stürzen, Frakturen und vorzeitigem Tod (auch durch kardiovaskuläre Erkrankungen). Neuere Untersuchungen belegen hier ebenfalls die Bedeutung einer kombinierten Vitamin-D- und Magnesiumtherapie (Abb. 3). Bei Vitamin-Dund Magnesiummangel wird die tägliche Substitution von Vitamin D (z.B. 1000 IE/Tag) und Magnesium (ca. 300-500 mg Mg/täglich in oraler Form) empfohlen. Hierbei zeigt sich ein signifikant positiver Effekt auf die Hypertonie [26].

\section{Vitamin K}

Ein erniedrigter Vitamin-K-Spiegel ist mit Hypertonie assoziiert, insbesondere in Kombination mit niedrigen Vitamin-D-Konzentrationen [27]. Bei Hypertonie-Patienten erhöht ein Vitamin-K-Mangel das Risiko für kardiovaskuläre Erkrankungen [28]. Positive Wechselwirkungen von Vitamin $\mathrm{D}$ und $\mathrm{K}$ werden in Abbildung 4 aufgezeigt. Ob eine Vitamin-K-Supplementation einen positiven Einfluss bei Hypertonie zeigt, ist derzeit nicht untersucht.

\section{Fazit}

Änderungen der Ernährungsgewohnheiten können einen wichtigen Beitrag bei der Behandlung von Bluthochdruck leisten. Insbesondere eine kochsalzarme Ernährung sowie eine kalium- und magnesiumreiche Kost 
Tab. 5. Vitamine und Hypertonie

\begin{tabular}{ll}
\hline Vitamin A & oxidativer Stress \\
Vitamin B & neurotrope Effekte \\
Vitamin C & oxidativer Stress \\
Vitamin D & Blutdruck \\
Vitamin E & oxidativer Stress \\
Vitamin K & Gefässverkalkung \\
\hline
\end{tabular}

wirken sich häufig positiv auf die Blutdrucksenkung aus. Erniedrigte Vitamin-D-Spiegel erhöhen das Risiko für
Hypertonie, während eine ausreichende Zufuhr das Mortalitätsrisiko im Alter senkt.

Eine spezifische Supplementation mit Elektrolyten und Vitaminen bei Hypertonie kann den Verlauf positiv beeinflussen. Besonders als adjuvante Therapie kann die pharmakologische Therapie mit klassischen Antihypertensiva verbessert und die Dosierungen und Nebenwirkungen reduziert werden.

\section{Literatur}

1 Neuhauser H, Diederichs C, Boeing H, et al: Bluthochdruck in Deutschland. Daten aus sieben bevölkerungsbasierten epidemiologischen Studien (1994-2012). Dtsch Arztebl 2016;113 809-815.

2 Houston M: Nutrition and nutraceutical supplements for the treatment of hypertension: part I. J Clin Hypertens (Greenwich) 2013;15: 752-757.

3 Houston M: Nutrition and nutraceutical supplements for the treatment of hypertension: part II. J Clin Hypertens (Greenwich) 2013;15: 845-851.

4 Houston M: Nutrition and nutraceutical supplements for the treatment of hypertension: part III. J Clin Hypertens (Greenwich) 2013; 15:931-937.

5 Lameris AL, Monnens LA, Bindels RJ, Hoenderop JGJ: Drug-induced alterations in $\mathrm{Mg} 2+$ homoeostasis. Clin Sci (Lond) 2012;123:1-14.

6 Graudal N, Jürgens G, Baslund B, Alderman $\mathrm{MH}$ : Compared with usual sodium intake, low and excessive sodium diets are associated with increased mortality. A meta-analysis. Am J Hypertens 2014;27:1129-1137.

7 Cappuccio FP, Markandu ND, Carney C, Sagnella GA, MacGregor GA: Double-blind randomized trial of modest salt restriction in older people. Lancet 1997;350:850-854.

8 He FJ, MacGregor GA: How far should salt intake be reduced? Hypertension 2003;42:10931099.

9 Zidek W: Kochsalz und Hypertonie. Cardiovasc 2013;1:49-50.

10 He FJ, Li J, Macgregor GA: Effect of longer term modest salt reduction on blood pressure: Cochrane systematic review and meta-analysis of randomised trials. BMJ 2013;346:f1325.

11 Houston M: The role of magnesium in hypertension and cardiovascular disease. J Clin Hypertens (Greenwich) 2011;13:843-847.

12 Poorolajal J, Zeraati F, Soltanian AR, Sheikh V, Hooshmand E, Maleki A: Oral potassium supplementation for management of essential hypertension: a meta-analysis of randomized controlled trials. PLoS One 2017;12:e174967.

Neben Elektrolyten und Vitaminen (Tab. 5) gewinnen auch weitere Nahrungsbestandteile wie Quercetin, pflanzliche Extrakte sowie OmegaFettsäuren zunehmend an Bedeutung bei der nichtmedikamentösen Therapie von Hypertonie [29, 30]. Dies sollte ebenso bezüglich der Ernährung von Hypertonikern Berücksichtigung finden.
13 Whelton SP, Blumenthal RS: Beyond the headlines: insights on potassium supplementation for the treatment of hypertension from the Canadian Hypertension Education Program guidelines (CHEP). Circulation 2017;135:3-4.

14 Zhang X, Li Y, Del Gobbo LC, Rosanoff A, Wang J, Zhang W, Song Y: Effects of magnesium supplementation on blood pressure: a meta-analysis of randomized double-blind placebo-controlled trials. Hypertension 2016; 68:324-333.

15 Kisters K, Gremmler B, Gröber U, Tokmak F: Magnesium metabolism, vitamin D and interleukins in cardiovascular disease. Metabolomics 2016,6:177.

16 Guerrero-Romero F, Rodríguez-Morán M, Hernández-Ronquillo $\mathrm{G}$, et al: Low serum magnesium levels and its association with high blood pressure in children. J Pediatr 2016;168: 93-98.e1.

17 Rosanoff A: Magnesium supplements may enhance the effect of antihypertensive medication in stage 1 hypertensive subjects. Magnes Res 2010;23:27-40.

18 Kienreich K, Tomaschitz A, Verheyen N, Pieber TR, Pilz S: Vitamin D and arterial hypertension: treat the deficiency! Am J Hypertens 2013;26:158.

19 Belen E, Sahin I, Güngör B, et al: Assessment of 25-hydroxyvitamin D levels in patients with resistant hypertension. Med Princ Pract 2016; 25:25-30.

20 Arora P, Song Y, Dusek J, et al: Vitamin D therapy in individuals with prehypertension or hypertension: the DAYLIGHT trial. Circulation 2015;131:254-262.

21 Pilz S, Gaksch M, Kienreich K, et al: Effects of vitamin $\mathrm{D}$ on blood pressure and cardiovascular risk factors: a randomized controlled trial. Hypertension 2015;65:1195-1201.

22 Tamez H, Kalim S, Thadhani RI: Does vitamin D modulate blood pressure? Curr Opin Nephrol Hypertens 2013;22:204-209.

23 Qi D, Nie X, Cai J: The effect of vitamin D supplementation on hypertension in nonCKD populations: a systemic review and meta-analysis. Int J Cardiol 2017;227:177-186.
24 Al Mheid I, Quyyumi AA: Vitamin D and cardiovascular disease: controversy unresolved. J Am Coll Cardiol 2017;70:89-100.

25 Bjelakovic G, Gluud LL, Nikolova D, Whitfield K, Wetterslev J, Simonetti RG, Bjelakovic M, Gluud C: Vitamin D supplementation for prevention of mortality in adults. Cochrane Database Syst Rev 2014;(1):CD007470.

26 Holick MF, Binkley NC, Bischiff-Ferrari HA, Gordon CM, Hanley DA, Heaney RP, Murad MH, Weaver CM; Endocrine Society: Evaluation, treatment and prevention of vitamin D deficiency: an Endocrine Society clinical practice guideline. J Clin Endocrinol Metab 2011; 96:1911-1930.

27 van Ballegooijen AJ, Cepelis A, Visser M, Brouwer IA, van Schoor NM, Beulens JW: Joint association of low vitamin D and vitamin $\mathrm{K}$ status with blood pressure and hypertension. Hypertension 2017;69:1165-1172.

28 Shea MK, Booth SL, Weiner DE, Brinkley TE, Kanaya AM, Murphy RA, Simonsick EM, Wassel CL, Vermeer C, Kritchevsky SB; Health ABC Study: Circulating vitamin $\mathrm{K}$ is inversely associated with incident cardiovascular disease risk among those treated for hypertension in the Health, Aging, and Body Composition Study (Health ABC). J Nutr 2017;147:888-895.

29 Serban MC, Sahebkar A, Zanchetti A, Mikhailidis DP, Howard G, Antal D, Andrica F, Ahmed A, Aronow WS, Muntner P, Lip GY, Graham I, Wong N, Rysz J, Banach M; Lipid and Blood Pressure Meta-analysis Collaboration (LBPMC) Group: Effects of quercetin on blood pressure: a systematic review and metaanalysis of randomized controlled trials. J Am Heart Assoc 2016;5:e002713.

30 Biesinger S, Michaels HA, Quadros AS, Qian Y, Rabovsky AB, Badger RS, Jalili T: A combination of isolated phytochemicals and botanical extracts lowers diastolic blood pressure in a randomized controlled trial of hypertensive subjects. Eur J Clin Nutr 2016;70:10-16. 